\title{
Análisis de la producción científica y de la especialización temática de la Universidad Politécnica de Valencia. Scopus (2003-2010)
}

\author{
María Adelina Codina-Canet*; Carlos Olmeda-Gómez*, Antonio Perianes-Rodríguez* \\ *Departamento de Biblioteconomía y Documentación. Universidad Carlos III de Madrid \\ Correo e: mariadelina.codina@alumnos.uc3m.es; carlos.olmeda@uc3m.es, antonio.perianes@ uc3m.es
}

Recibido:05-02-2012; 2a versión:02-07-2012; Aceptado: 03-07-2012

Cómo citar este artículo/ Citation: Codina-Canet, M. A.; Olmeda-Gómez, C.; Perianes-Rodríguez, A. (2013). Análisis de la producción científica y de la especialización temática de la Universidad Politécnica de Valencia. Scopus (2003-2010). Revista Española de Documentación Científica, 36(3):e019. doi: http://dx.doi.org/10.3989/redc.2013.3.942

Resumen: Se analiza la producción científica con visibilidad internacional de la Universidad Politécnica de Valencia (UPV) recogida en las bases de datos de SCOPUS durante el período 2003-2010. Los 11.126 documentos publicados representan el $23 \%$ de la producción valenciana y el 2,8\% de la producción española. La caracterización de la especialización temática y su evolución a lo largo del período analizado muestra que más de la mitad de la producción se adscribe a las áreas de Ingeniería, Informática, Química y Matemáticas, mostrándose como las principales áreas del desarrollo científico institucional. Además, un tercio de los trabajos se publican en forma de comunicaciones a congresos. Si a ello se unen los publicados en forma de artículos, ambas tipologías representan el 95\% de la producción. A su vez, el 95\% de los trabajos se publican en inglés y reciben más del $99,5 \%$ de las citas totales.

Por otro lado, y derivado de su comparación con el resto de politécnicas nacionales, la UPV es la segunda en producción y citación absolutas, por detrás de la de Cataluña. Mantiene una posición predominante en Agricultura, Biología Molecular, Química, Ciencias de la Decisión, Ciencias Sociales y Química. Aunque sus principales especialidades son de carácter politécnico, son otras áreas menos técnicas las que presentan mayor representatividad y visibilidad, evidenciando señas de identidad distintivas respecto de sus homólogas.

Por último, y desde la perspectiva del impacto, el retroceso observado de la visibilidad esperada se corresponde con un aumento de los trabajos publicados en revistas de tercer y cuarto cuartil. A pesar de ese descenso, la UPV cuenta con visibilidades esperadas superiores a las obtenidas por las principales politécnicas nacionales.

Palabras clave: Producción Científica; especialización temática; educación Superior; Universidad Politécnica de Valencia; Evaluación de la Investigación; Scopus.

Analysis of scientific production and research specialization of the Universidad Politécnica de Valencia. Scopus (2003-2008)

Abstract: This study analyzes the scientific output and research specialization at the Universidad Politécnica de Valencia (UPV) as collected by the SCOPUS database during 2003-2010. The 11,126 documents published represent $23 \%$ of the regional production and $2.8 \%$ of Spanish production. The analysis of the evolution of subject specialization shows that more than half of the production is based on Engineering, Computer Science, Chemistry and Mathematics, as the main areas. A third of the works were published as conference papers. Furthermore $95 \%$ of the papers were published in English and received more than $99 \%$ of the overall citations. 
The comparison with other national polytechnics shows that the UPV is second only to the Univ. of Politécnica de Cataluña in production and citation. It maintains a dominant position in Agriculture, Biology, Chemistry, Decision Sciences, Social Sciences and Chemistry. Although its main specialties are technical, the other less technical fields are more representative with a higher visibility, thereby confirming its distinctive profile.

Finally, the impact analysis shows that the decrease in expected visibility corresponds to an increasing number of papers published in journals of the third and fourth quartiles. Despite this decline, the UPV has a higher citation rate than that of the other Spanish polytechnic universities.

Keywords: Scientific production; research specialties; higher Education; Universidad Politécnica de Valencia; research evaluation; Scopus.

Copyright: (C) 2013 CSIC. Este es un artículo de acceso abierto distribuido bajo los términos de la licencia Creative Commons Attribution-Non Commercial (by-nc) Spain 3.0

\section{INTRODUCCIÓN}

La Universidad Politécnica de Valencia ha evolucionado desde su creación en 1971 hacia una labor integradora, desarrollando un modelo que intenta armonizar el carácter social con el emprendedor, para contribuir a la consolidación de la ciencia y la tecnología en el sistema sociocultural valenciano, redundando en un aumento de la calidad de su enseñanza y su investigación (Reyes Davó, 1994). Con esta vocación de servicio a la sociedad y al desarrollo empresarial, puso en marcha en 2002 el parque científico "Ciudad Politécnica de la Innovación" (CPI), con el fin de estrechar la colaboración entre institutos de investigación, empresas y otras instituciones emprendedoras e innovadoras según un modelo de Red de Colaboración Abierta, enfatizando la importancia de la transferencia tecnológica a la sociedad.

La Universidad Politécnica de Valencia, en el componente científico técnico organiza su actividad investigadora y de transferencia tecnológica a través de estructuras de investigación: departamentos universitarios, institutos de investigación, estructuras propias de investigación (EPI), grupos de I+D+i y estructuras de apoyo.

En el curso 2010/11 contaba con 42 departamentos y 15 institutos universitarios dedicados a la investigación científica, técnica y artística, de carácter propio o mixto, creados en colaboración con organismos públicos o privados mediante convenios de asociación. Los institutos son los centros que agrupan a mayor número de investigadores y tienen un papel destacado en la actividad productiva. Once son propios y cuatro mixtos. Los grupos de $I+D+i$, se caracterizan por líneas de investigación específicas y una oferta tecnológica propia. Actualmente en la UPV desarrollan su labor de investigación cerca de 350 grupos registrados. A ello hay que sumar 14 centros de investigación y 3 centros en red. También dispone de estructuras de apoyo como el Centro de Apoyo a la Innovación, la Investigación y la Transferencia de Tecnología (CTT), o los Servicios de Microscopía Electrónica y Radiaciones. EI CTT depende jerárquicamente del Vicerrectorado de Investigación, Desarrollo e Innovación y colabora estrechamente con el Área de Planificación, Evaluación e Iniciativas de Investigación, tratando de integrar los distintos Institutos, Centros y Grupos dedicados a la investigación.

El vigente Plan Estratégico UPV 2007/2014 prevé la ampliación y consolidación de las estructuras de $\mathrm{I}+\mathrm{D}+\mathrm{i}$ de la UPV en grandes proyectos de investigación, con el propósito de incrementar su participación en proyectos estratégicos (nacionales e internacionales) de gran tamaño, fomentando la creación de consorcios ad hoc con institutos y empresas (UPV, 2007).

El grueso del componente científico-técnico de la Universidad Politécnica de Valencia participa en la CPI, canalizando su actividad investigadora y de transferencia tecnológica a través de centros e institutos de investigación. La estructura organizativa se amplió en 2011 gracias a su participación en el Campus de Excelencia Internacional (VLC/ CAMPUS), junto con la Universitat de València y el Consejo Superior de Investigaciones Científicas (CSIC). VCL/CAMPUS organiza sus recursos en Micro-Clusters de Investigación (MCI) interdisciplinares e interuniversitarios. Actualmente cuenta con $18 \mathrm{MCI}$ identificados que trabajan en nuevos enfoques conceptuales en Salud, Información y Comunicación, y Sostenibilidad (UPV, 2011a; 2011b).

En este sentido, y al amparo de este contexto institucional, son necesarios los estudios que ayu- 
den a conocer la evolución de los resultados de investigación de la UPV, y la determinación de los dominios preferentes de especialización de su producción científica. Por ello, proponemos un trabajo para el análisis de las fortalezas de la investigación en esta institución, con el fin de establecer el patrón de crecimiento y especialización de su producción científica, tomando como punto de partida artículos precedentes, y los resultados de la valoración de la actividad investigadora de las estructuras de investigación de la propia Universidad (Alonso-Arroyo y otros, 2005; 2006; UPV, 2011c).

\section{OBJETIVOS}

El objetivo principal es el análisis global y la evaluación de la actividad investigadora con visibilidad internacional, y de los dominios científicos de especialización de los 2778 docentes e investigadores de la UPV, en el período 2003-2010, dando respuesta a preguntas de investigación como:

- ¿Cuál es la aportación de la UPV a la producción global de la Comunitat Valenciana y a España?

- ¿Cuál es la distribución temática de la producción científica de la UPV?

- ¿Es distintivo su perfil temático respecto a los dominios geográficos de referencia (nacional y regional)? ¿Y respecto al resto de universidades politécnicas nacionales?

- ¿Cuáles son los hábitos de publicación de los investigadores en revistas con orientación internacional? ¿Y las tipologías documentales que utilizan para difundir sus estudios?

- ¿Cómo evolucionan los indicadores de visibilidad de la producción de la UPV?

Este trabajo trata de servir como una herramienta de información para el análisis de la producción científica institucional, cuyos resultados puedan contrastarse con los hallazgos realizados en otros estudios, y como instrumento de ayuda adicional en la toma de decisiones institucional.

\section{MATERIAL Y MÉTODOS}

Está universalmente aceptado que las revistas científicas son el principal vehículo para la transmisión de los resultados de investigación y su plasmación en forma de publicación. Los recuentos de los trabajos publicados en esas revistas son una operación aditiva que proporciona la información necesaria, partiendo de la base de que todas las publicaciones son equiparables como portadoras de conocimiento científico (Maltrás, 2003; Sancho, 2002). En este sentido, y a pesar de los esfuerzos del personal bibliotecario y del departamento afín de la universidad en la elaboración del repositorio científico institucional, la realización de un análisis exhaustivo pasa por el acceso a las bases de datos de las editoriales y revistas científicas, gracias a los servicios de valor añadido que estas presentan. Estas características singulares son actualmente exclusivas de estos productos, por lo que son considerados las fuentes más fiables para este tipo de estudios. Sin embargo, es preciso recordar que las grandes bases de datos (Scopus, Web of Science), ofrecen una visión general de la producción investigadora desde una perspectiva internacional, por lo que se presentan como instrumentos idóneos para la aproximación a este tipo de análisis.

La fuente de datos utilizada ha sido el portal SCImago Institutions Ranking (SIR), que analiza la producción científica de más de 3000 instituciones internacionales (SIR, 2012a). La fuente de datos del SIR es Scopus, recurso cuyas ventajas y bondades para este tipo de estudios han sido analizadas y puestas de manifiesto en trabajos como los de Goodman y Deis (2005), LaGuardia (2005), BarIlan (2010), Escalona y otros (2011), y Leydesdorff (2012), entre otros. Tradicionalmente, la fuente de información utilizada en estudios precedentes ha sido el conjunto de bases de datos recogidas en Web of Science (WoS) de Thomson Reuters. Entre las principales ventajas que ofrece su uso se encuentra su carácter multidisciplinar e internacional. Estas bases recogen información de 9.000 de las principales revistas científicas, y se han constituido en la herramienta de referencia mundial para la recuperación de información y para los estudios de evaluación de la ciencia. Sin embargo, la aparición en el mercado de nuevas herramientas y proyectos permite realizar análisis más exhaustivos de los resultados de la actividad científica internacional. Tal es el caso de la base de datos Scopus desarrollada por Elsevier, permitiendo ampliar los análisis obtenidos a partir de los productos de Thomson Reuters. Las razones del uso de esta nueva base de datos tienen que ver con la mayor cobertura de la fuente (más de 15.000 revistas), que supone una mejor representación de la ciencia española en el nivel internacional y por extensión, de la producción científica de la UPV. Por lo que respecta a la cobertura temática, Elsevier ha hecho especial hincapié en las áreas de Ciencias, Tecnología y Medicina, y en Ciencias Sociales en las áreas de Psicología, Sociología y Economía. Excepto para el caso de Lengua, Literatura, Filosofía y Teología, Scopus presenta una mejor cobertura que WoS (Bosman y otros, 2006). Por tanto, nos encontramos ante una herramienta multidisciplinar, internacional y con una fuerte orientación tecnológica, lo que permite ampliar estudios anteriores en los que la cobertura temática en este sentido se erigía como una limitación. Consecuentemente, el universo de citación de esta fuente es también superior, debido a la mayor presencia de documentos citables.

Por otro lado, es preciso aclarar que cuando se analizan los datos desagregados en las distintas categorías temáticas en las que Scopus divide el conocimiento científico, es inherente la obtención de duplicidades. Esa doble contabilidad es conse- 
cuencia del sistema utilizado para la agrupación de las revistas científicas, basado en la asignación de la especialidad según la de la revista de origen. En concreto, Scopus clasifica las revistas en 295 categorías temáticas que posteriormente se agrupan en 27 grandes áreas de conocimiento. Dado que es frecuente que los títulos de origen contengan más de una categoría temática, el recuento provoca solapamiento, que en el caso de la UPV asciende a 3059 documentos sobre la producción real de 11126 .

Una vez obtenidos los datos se procedió al análisis descriptivo de las áreas temáticas, obteniéndose una triple dimensión en orden decreciente basada en la producción científica, la citación esperada y observada, y la tasa de crecimiento. Esas tres dimensiones permiten clasificar los dominios según su grado de consolidación en el período, facilitando tanto la descripción, como la caracterización de la especialización científica de la UPV (incluyendo los centros mixtos con el CSIC).

Para conocer la representatividad de cada área temática en la institución, se calcularon adicionalmente valores de producción y porcentajes de contribución anual de cada especialidad.

En cuanto a la tasa de crecimiento de la producción por áreas, se han tenido en cuenta las áreas más representativas, excluyendo aquéllas con escasa significación (menos de 10 documentos anuales).

\subsection{Indicadores}

Los principales indicadores utilizados han sido:

\section{a) Cuantitativos:}

- Producción absoluta: señala el número de documentos de cualquier tipo en los que interviene al menos un autor adscrito a la institución. La segregación por áreas temáticas de la producción total dificulta la realización de comparaciones, por cuanto tanto los entornos como las propias características de los ciclos productivos de cada disciplina afectan de forma considerable en los resultados finales. La identificación de los autores se realiza mediante la comprobación sistemática de su pertenencia a la UPV a través de la filiación institucional recogida en el campo correspondiente del registro de la base de datos.

- Tasa de crecimiento: muestra el aumento productivo que el dominio (institución/región) presenta respecto al año anterior. Es, por tanto, la diferencia porcentual del número de trabajos en relación con el período anterior. Su cálculo anual permite calibrar la evolución del agregado a lo largo del período analizado.

\section{b) De visibilidad:}

- Citación absoluta (observada): por visibilidad se entiende el impacto de cada publicación medido a partir del número real de citas recibidas por un trabajo. Esto permite analizar la repercusión que la difusión del conocimiento científico logra en la comunidad científica en todos los niveles de agregación y cuya unidad de análisis es la cita bibliográfica. Este indicador absoluto decrece a medida que se aproxima al presente, sirviendo de ejemplo para el proceso de uso y consumo de la información. La inclinación de la curva descendente dependerá en gran medida de los hábitos de publicación del área.

- Citas por documento: presenta el promedio de citas recibidas por el total de la producción científica. Permite relativizar los tamaños ponderando las dos dimensiones: cantidad y visibilidad.

- ASSJR (citación esperada): es el promedio del SCImago Journal Rank normalizado (González-Pereira y otros, 2009). Si un trabajo presenta varios SSJR, como consecuencia de su adscripción a diferentes categorías, se empleará el mayor de los valores.

Este trabajo se centra exclusivamente en los resultados obtenidos a partir de las investigaciones científicas publicadas, es decir, sólo analiza aquellos resultados que utilizan las revistas científicas como vehículo de comunicación. Quedan, por tanto, excluidos otros resultados propios de la investigación y la innovación tecnológica. La evaluación exclusiva de los resultados publicados en canales formales internacionales es una limitación inherente a los indicadores bibliométricos, puesto que estos descartan cualquier otra forma de expresión científica. Sin embargo, la literatura internacional es una muestra suficientemente significativa, ya que la mayor parte de lo que circula en circuitos informales termina publicándose en canales formales y, aunque esto no sucede siempre, la confirman como un ejemplo representativo de la actividad científica de ámbito internacional. Pese a todo, la evaluación a través de indicadores bibliométricos es tremendamente útil. Disponer de indicadores, estudiar sus sesgos y minimizar sus efectos negativos, debe ser una constante. La mayor parte de los problemas pueden ser minimizados utilizando una gran variedad de indicadores y éstos, a su vez, deben ser complementados y contrastados con otro tipo de análisis y perspectivas, como el sistema de revisión por expertos, los retornos económicos generados por la investigación o la capacidad tecnológica adquirida, entre otros.

\section{RESULTADOS}

\subsection{Producción y citación de la UPV respecto a España y Valencia}

Como ya se ha indicado, las publicaciones con visibilidad internacional de la UPV ascienden a 11126 , y representan el $22,9 \%$ de la producción 
absoluta de las instituciones valencianas. Todas ellas, a su vez, suponen el $12,3 \%$ de la producción española, por lo que la UPV aporta el 2,8\% al total nacional. La tabla I muestra los datos interanuales globales de producción, citación y tasa de crecimiento en España, Valencia (Comunitat) y UPV. En el caso de la producción científica de la Universidad, se observa que los años con mayor aportación al total regional fueron 2009 y 2010 , con el $26,4 \%$ y $25,7 \%$. Entre 2004 y 2008 su aportación se sitúa entre el 21 y el $23 \%$, mientras que en 2003 representa el $18 \%$ de las publicaciones valencianas.

Por lo que respecta a las citas, la UPV representa el $17,7 \%$ de la visibilidad total de la Comunitat Valenciana. 2009 y 2010 son los años con mayor aportación, en torno al 19\%. La visibilidad observada de la UPV respecto a Valencia es, por tanto, ligeramente inferior a la representatividad que muestra su producción científica.

La figura 1 presenta la evolución del crecimiento de la producción. Se observa una evolución dife- rente a la de sus dominios geográficos de referencia, caracterizada por un marcado descenso en el primer tercio del período, al que sigue una evolución alterna de aumentos y reducciones que concluye con un final de ciclo sin crecimiento. Como contraste, la Comunitat Valenciana presenta un descenso continuo desde 2005 y España un tímido crecimiento desde 2006.

Nótese cómo el incremento de las publicaciones de la UPV en 2005-2006 ascendió a 142 documentos, representando una aportación cercana al $18 \%$ del crecimiento de la producción regional, bajo porcentaje compensado en 2006-2007 con un incremento de 268 trabajos, que representan casi el $50 \%$ del crecimiento producido en la Comunitat Valenciana, que para ese intervalo representa un crecimiento de 547 artículos. Sin embargo en el período posterior (2007-2008) la UPV aporta apenas el $11 \%$ a la Comunitat.

Tabla I. Producción científica y tasa de crecimiento comparada. 2003-2010

\begin{tabular}{|c|c|c|c|c|c|c|c|c|c|}
\hline & \multicolumn{3}{|c|}{ ESP } & \multicolumn{3}{|c|}{ VAL } & \multicolumn{3}{|c|}{ UPV } \\
\hline & Pc & $\mathbf{C i}$ & TC Esp & Pc & $\mathbf{C i}$ & TC Val & Pc & $\mathbf{C i}$ & TC UPV \\
\hline 2003 & 35293 & 559035 & & 4154 & 71929 & & 760 & 12380 & \\
\hline 2004 & 37934 & 539373 & 0,07 & 4639 & 69779 & 0,12 & 976 & 11823 & 0,28 \\
\hline 2005 & 43214 & 531855 & 0,14 & 5263 & 69759 & 0,13 & 1143 & 12149 & 0,17 \\
\hline 2006 & 47897 & 496084 & 0,11 & 6129 & 64557 & 0,16 & 1285 & 11650 & 0,12 \\
\hline 2007 & 50649 & 439995 & 0,06 & 6676 & 54261 & 0,09 & 1553 & 10093 & 0,21 \\
\hline 2008 & 54716 & 344016 & 0,08 & 7056 & 48560 & 0,06 & 1597 & 8065 & 0,03 \\
\hline 2009 & 60215 & 231183 & 0,10 & 7228 & 29437 & 0,02 & 1906 & 5804 & 0,19 \\
\hline 2010 & 65095 & 96460 & 0,08 & 7427 & 11342 & 0,03 & 1906 & 2146 & 0 \\
\hline Total & 395013 & 3238001 & 0,84 & 48572 & 419624 & 0,79 & 11126 & 74110 & 1,51 \\
\hline
\end{tabular}

Pc Producción citable, $\mathrm{Ci}=$ Citación, $\mathrm{TC}=$ Tasa de Crecimiento

Fuente: SCImago Institutions Ranking

Figura 1. Evolución de la tasa de crecimiento de la producción comparada. 2003-2010

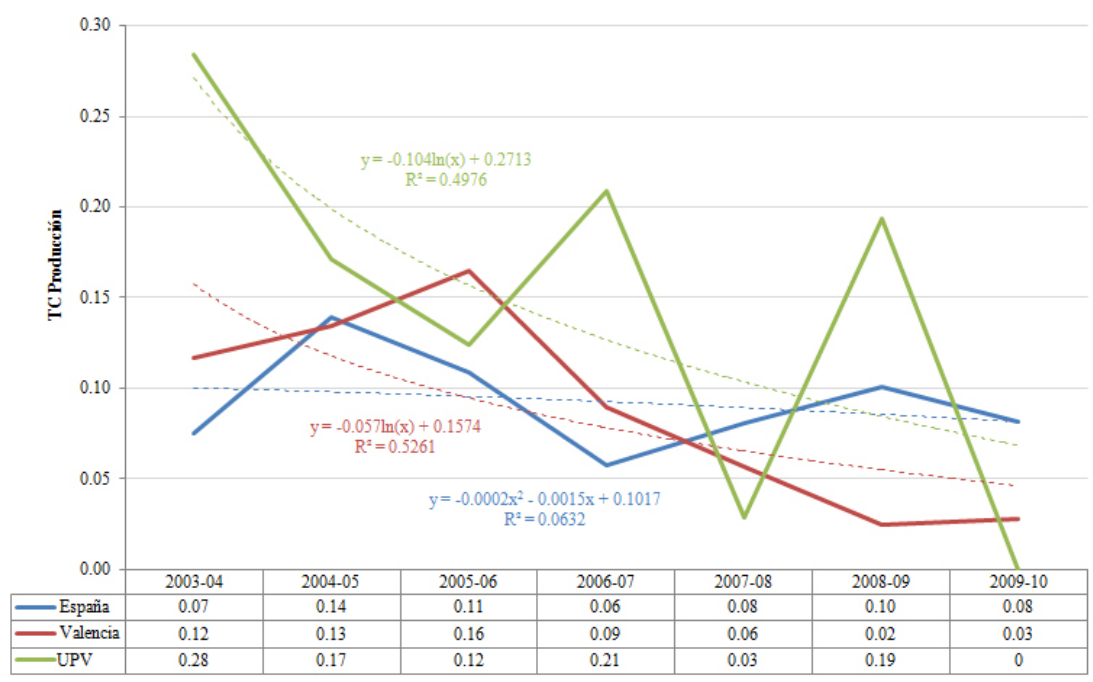


Las tablas II y III muestran la distribución de la producción por idiomas y tipos documentales durante el período.

La figura 2 presenta la distribución porcentual anual de los trabajos de los investigadores de la UPV según el cuartil de la revista en los que han sido publicados. Destaca el crecimiento de los trabajos publicados en tercer y cuarto, que son los encargados de recibir los diez puntos de diferencia que pierden los del segundo. El volumen absoluto de trabajos en el primer cuartil es importante y su valor porcentual se mantiene constante en torno al $40 \%$.

Tabla II. Distribución de la producción por idiomas. UPV 2003-2010

\begin{tabular}{|c|c|c|c|c|}
\hline Idioma & Pc & $\mathbf{C i}$ & $\%$ Pc & $\% \mathbf{C i}$ \\
\hline Inglés & 10703 & 73834 & 95,94 & 99,56 \\
\hline Español & 427 & 294 & 3,83 & 0,40 \\
\hline Francés & 10 & 13 & 0,09 & 0,02 \\
\hline Portugués & 9 & 15 & 0,08 & 0,02 \\
\hline Alemán & 3 & 0 & 0,03 & 0 \\
\hline Ruso & 2 & 0 & 0,02 & 0 \\
\hline Japonés & 1 & 0 & 0,01 & 0 \\
\hline Lituano & 1 & 1 & 0,01 & 0,001 \\
\hline
\end{tabular}

Pc Producción citable, $\mathrm{Ci}=$ Citación

Fuente: SCImago Institutions Ranking

Tabla III. Distribución de la producción por tipos documentales. UPV 2003-2010

\begin{tabular}{ccccccccccccc}
\hline & Art & Com & Rev & Ed & Err & Let & Not & Sur & Pres & Total \\
\hline 2003 & 544 & 177 & 30 & 2 & 2 & 2 & 1 & 2 & 0 & 760 \\
\hline 2004 & 638 & 313 & 21 & 0 & 1 & 2 & 0 & 1 & 0 & 976 \\
\hline 2005 & 657 & 432 & 36 & 5 & 3 & 4 & 5 & 1 & 0 & 1143 \\
\hline 2006 & 808 & 433 & 30 & 5 & 1 & 3 & 4 & 1 & 0 & 1285 \\
\hline 2007 & 971 & 541 & 25 & 8 & 3 & 1 & 3 & 1 & 0 & 1553 \\
\hline 2008 & 1007 & 517 & 25 & 13 & 3 & 6 & 14 & 9 & 1597 \\
\hline 2009 & 1252 & 576 & 32 & 12 & 5 & 1 & 12 & 6 & 9 \\
\hline 2010 & 1147 & 647 & 37 & 15 & 4 & 2 & 9 & 1 & 43 \\
\hline TOTAL & 7024 & 3636 & 236 & 60 & 22 & 21 & 48 & 16 & 61 & 11124 \\
\hline
\end{tabular}

Art: Artículo. Com: Comunicación. Rev: Revisión. Ed: Editorial. Err: Errata. Let: Carta. Not: Nota. Sur: Encuesta. Pres: En prensa Fuente: SCImago Institutions Ranking

Figura 2. Distribución porcentual de producción por cuartiles. UPV

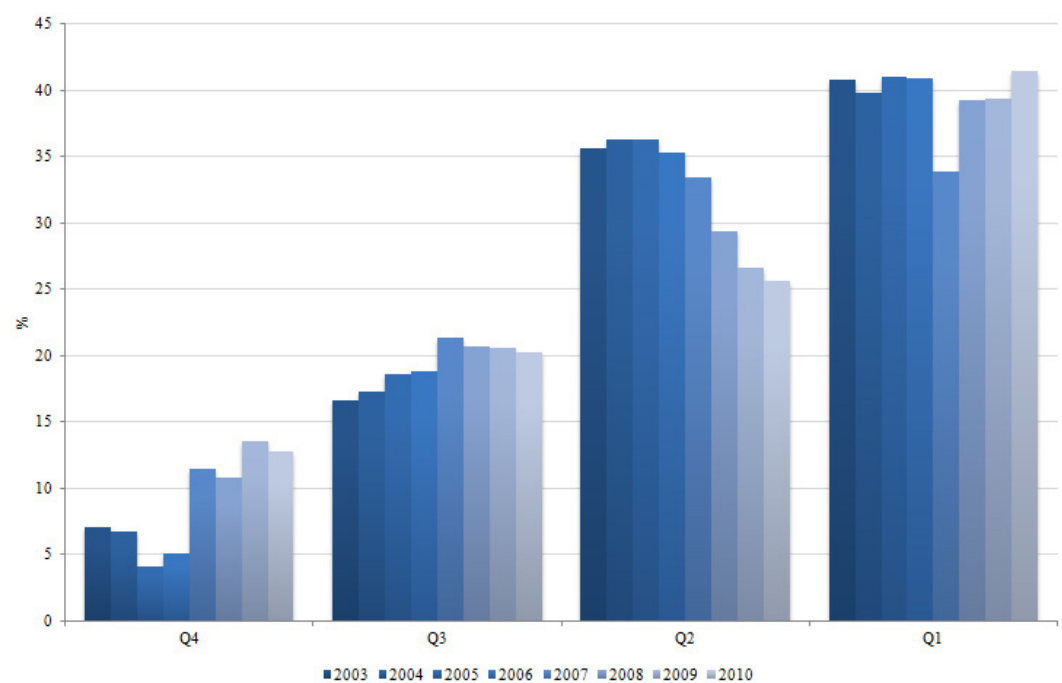




\subsection{Producción y citación respecto a las uni- versidades politécnicas nacionales}

La UPV ocupa la segunda posición de las universidades politécnicas españolas en producción y citación absolutas, solo superada por la Universitat Politécnica de Cataluña (UPC).

La comparación de la producción de la UPV con las otras tres politécnicas permite analizar con mayor detalle su representatividad y visibilidad descendiendo al nivel de área temática. Así, y por lo que respecta a la productividad, es la primera en áreas como Agricultura, Biología Molecular, Inmunología,
Medicina o Química, con más del $40 \%$ de la producción politécnica nacional en cada una de ellas.

Trasladando la comparación a la visibilidad, la UPV mantiene su posición predominante en Agricultura y Biología Molecular. A pesar de que las disciplinas médicas no acompasan su representatividad con su visibilidad en esa primera posición, destaca la visibilidad de Ingeniería Química, superior al $50 \%$ del global politécnico, pero especialmente Química, con más del $70 \%$ de las citas totales de las cuatro universidades. (Tabla IV)

Tabla IV. Producción y Citación de las Universidades Politécnicas Españolas. Áreas Temáticas. 2003-2010

\begin{tabular}{|c|c|c|c|c|c|c|c|c|}
\hline \multirow{2}{*}{ Área Temática } & \multicolumn{4}{|c|}{ Producción absoluta } & \multicolumn{4}{|c|}{ Citación absoluta } \\
\hline & UPV & UPC & UPM & UPCART & UPV & UPC & UPM & UPCART \\
\hline Agricultura & 1423 & 505 & 1166 & 375 & 11897 & 4136 & 9446 & 2698 \\
\hline Arte y Humanidades & 30 & 172 & 25 & 3 & 92 & 148 & 54 & 1 \\
\hline Biología Molecular & 594 & 376 & 328 & 42 & 7007 & 6422 & 2834 & 212 \\
\hline Empresa & 154 & 132 & 65 & 78 & 580 & 294 & 283 & 332 \\
\hline Ingeniería Química & 748 & 862 & 323 & 133 & 8409 & 5170 & 1484 & 877 \\
\hline Química & 1182 & 652 & 307 & 175 & 20752 & 4754 & 2088 & 1551 \\
\hline Informática & 2122 & 3413 & 1939 & 323 & 6972 & 16128 & 7074 & 1038 \\
\hline Ciencias de la Decisión & 244 & 195 & 130 & 28 & 1727 & 806 & 968 & 115 \\
\hline Odontología & 5 & 4 & 0 & 1 & 13 & 17 & 0 & 0 \\
\hline Ciencias de la Tierra & 134 & 1020 & 365 & 82 & 619 & 7474 & 1723 & 673 \\
\hline Economía & 52 & 73 & 49 & 60 & 283 & 281 & 115 & 207 \\
\hline Energía & 175 & 178 & 348 & 34 & 1008 & 1045 & 1879 & 141 \\
\hline Ingeniería & 2287 & 3801 & 2810 & 544 & 11497 & 21228 & 12888 & 2396 \\
\hline Ciencias Medioambientales & 449 & 728 & 478 & 103 & 2907 & 5986 & 3164 & 828 \\
\hline General & 60 & 53 & 62 & 11 & 1922 & 1883 & 1289 & 9 \\
\hline Profesiones Sanitarias & 38 & 25 & 40 & 5 & 335 & 334 & 342 & 22 \\
\hline Inmunología y Microbiología & 147 & 91 & 125 & 12 & 1635 & 925 & 1918 & 126 \\
\hline Ciencia de Materiales & 827 & 1284 & 786 & 148 & 6345 & 7556 & 4259 & 967 \\
\hline Matemáticas & 1702 & 2634 & 1348 & 337 & 5094 & 10689 & 3294 & 1004 \\
\hline Medicina & 510 & 315 & 370 & 95 & 2574 & 2173 & 2731 & 168 \\
\hline Neurociencias & 16 & 29 & 14 & 1 & 80 & 217 & 35 & 11 \\
\hline Enfermería & 5 & 5 & 7 & 2 & 15 & 20 & 47 & 7 \\
\hline Farmacología y Farmacia & 44 & 83 & 17 & 6 & 553 & 1206 & 175 & 90 \\
\hline Física y Astronomía & 886 & 1711 & 1329 & 143 & 6512 & 14168 & 7347 & 752 \\
\hline Psicología & 38 & 19 & 21 & 9 & 210 & 84 & 30 & 7 \\
\hline Ciencias Sociales & 264 & 232 & 221 & 37 & 1279 & 780 & 575 & 87 \\
\hline Veterinaria & 49 & 5 & 57 & 0 & 258 & 7 & 276 & 0 \\
\hline TOTAL & 14185 & 18597 & 12730 & 2787 & 100575 & 113931 & 66318 & 14319 \\
\hline
\end{tabular}

UPV: Universidad Politécnica de Valencia. UPC: Universidad Politécnica de Cataluña. UPM: Universidad Politécnica de Madrid. UPCART Universidad Politécnica de Cartagena

Fuente: SCImago Institutions Ranking 


\subsection{Producción científica por áreas temáti- cas. La especialización de la UPV}

El volumen de producción desagregado por áreas científicas no sólo refleja la actividad de las distintas disciplinas y su capacidad para generar conocimiento sino también la caracterización de la especialización temática institucional. Esta desagregación permite, además, la descripción de las áreas en la UPV, el análisis de su especialización tecnológica y la comparación con sus dominios geográficos de referencia, Valencia y España.

La distribución temática porcentual de la producción de la figura 3 compara los patrones de publicación regional y nacional, ambos muy similares, con el institucional, que presenta un patrón temático distintivo.

La UPV publicó 2287 documentos en Ingeniería, área más productiva y que representa el $16 \%$ de la producción institucional. Esos trabajos suponen el $56 \%$ de todos los realizados en Valencia, y el 6,5\% de los firmados en España. En valores similares a los de Ingeniería se encuentra la representatividad regional y nacional de Informática.

El hecho de tratarse de una universidad politécnica y dada su mayor dedicación a carreras técnicas, no sorprende que los resultados ofrezcan una posición institucional predominante en Valencia y España en disciplinas como Ingeniería, Informática, Matemáticas, Ingeniería Química o Ciencia de Materiales.

En cuanto al patrón de publicación de la UPV en el denominado triángulo de la Ciencia por Herrero y
Vargas, que distribuye los patrones de publicación en tres ejes temáticos; el primero se corresponde con el dominio de las ciencias biomédicas (Medicina, Bioquímica, Genética, Biología Molecular). En el segundo predominan las ciencias fundamentales (Física, Química, Matemáticas) y las ingenierías. En el tercero domina la Agricultura (Herrero y Vargas, 2010). Así, mientras que España y la Comunitat Valenciana se sitúan en el primero de los ejes, la producción de la UPV se encuadra en el segundo, con un perfil similar al de países de la Europa del Este, Rusia y las antiguas Repúblicas Soviéticas, en los que predominan la Física y las Matemáticas, y al de los países del sudeste asiático, con un marcado sesgo hacia las ingenierías.

La tabla $\mathrm{V}$ muestra los resultados de la tasa de crecimiento y el promedio de citas por documento de cada área.

El número medio de citas por documento es de 7,67 . Los valores superiores al promedio más destacados se registran en General con 32,03, seguida de Química con 17,56, Farmacología con 12,57 citas por documento.

\subsection{Caracterización de los principales domi- nios temáticos}

Este apartado amplía el análisis de las áreas científicas más relevantes según la producción científica con visibilidad internacional: Ingeniería, Informática, Matemáticas, Agricultura, Química, Ciencia de Materiales, Física, Ingeniería Química, y aquellas áreas que destacan de su comparación con el resto de universidades politécnicas españolas:

Figura 3. Distribución porcentual de la producción por dominios. Áreas temáticas. UPV 2003-2010

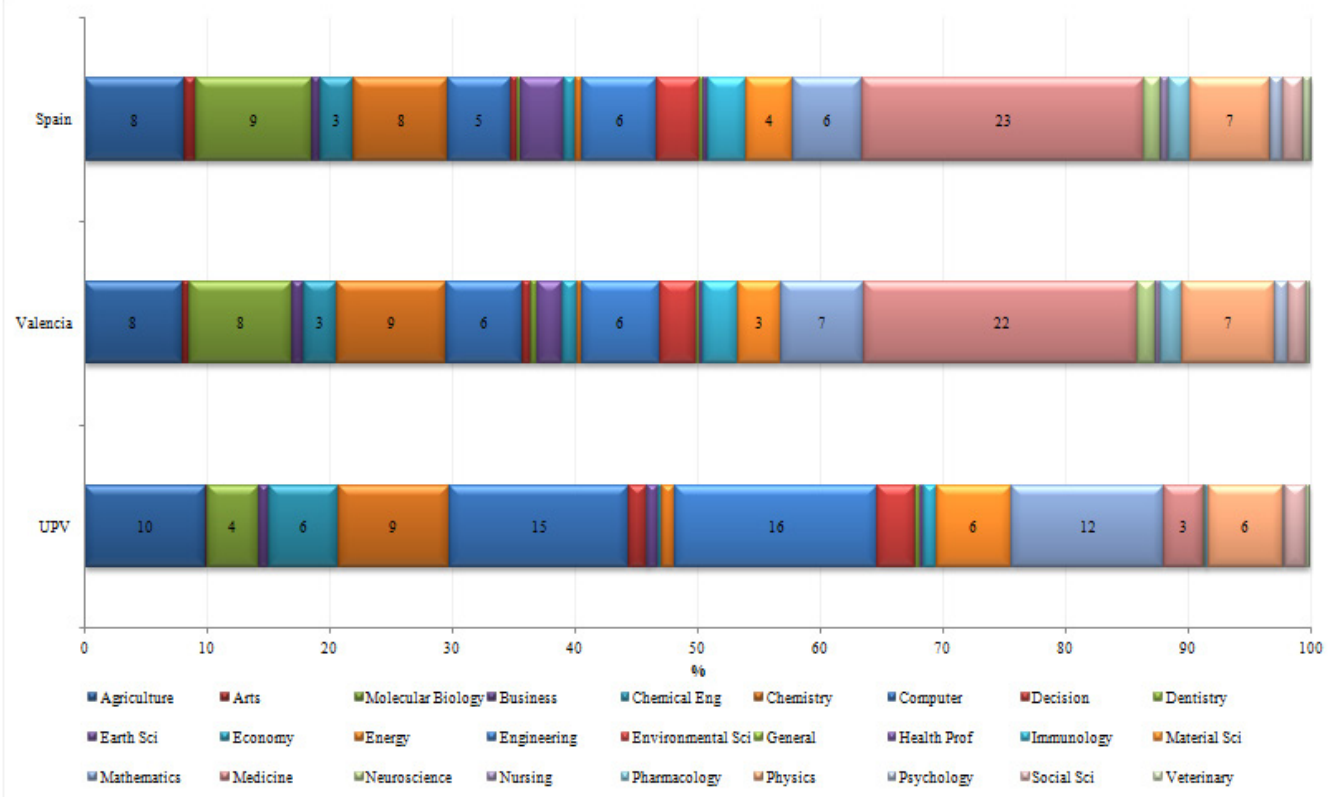


Tabla V. Producción Científica. Tasas de Crecimiento. Áreas Temáticas. UPV 2003-2010

\begin{tabular}{|c|c|c|c|c|c|c|c|c|c|}
\hline Área Temática & 03-04 & 04-05 & 05-06 & $06-07$ & 07-08 & 08-09 & 09-10 & Período & CpD \\
\hline Agricultura & $-0,13$ & 0,31 & 0,21 & 0,26 & 0,05 & 0,06 & 0,08 & 1,08 & 8,36 \\
\hline Arte y Humanidades & 1,00 & $-1,00$ & & 4,00 & $-0,20$ & 1,00 & 0,13 & 8,00 & 3,07 \\
\hline Biología Molecular & $-0,04$ & $-0,07$ & 0,33 & 0,24 & $-0,05$ & 0,33 & $-0,12$ & 0,63 & 11,8 \\
\hline Empresa & 0,29 & $-0,56$ & 4,25 & $-0,29$ & 0,87 & 0,25 & 0,00 & 4,00 & 3,77 \\
\hline Ingeniería Química & 0,59 & 0,13 & 0,09 & $-0,07$ & $-0,14$ & 0,35 & $-0,08$ & 0,96 & 11,24 \\
\hline Química & 0,64 & 0,21 & $-0,01$ & $-0,01$ & $-0,08$ & 0,17 & 0,03 & 1,16 & 17,56 \\
\hline Informática & 0,27 & 0,16 & $-0,12$ & 0,43 & 0,01 & 0,25 & 0,01 & 1,35 & 3,29 \\
\hline Ciencias de la Decisión & $-0,08$ & 0,50 & 0,39 & 0,08 & 0,52 & 0,10 & 0,40 & 3,85 & 7,08 \\
\hline Odontología & & & $-1,00$ & & & & 0,00 & & 2,6 \\
\hline Ciencias de la Tierra & 1,00 & 0,13 & $-0,39$ & 0,36 & 0,40 & 0,24 & $-0,27$ & 1,38 & 4,62 \\
\hline Economía & $-1,00$ & & 2,00 & $-0,17$ & 1,40 & $-0,25$ & 0,56 & 2,50 & 5,44 \\
\hline Energía & $-0,22$ & 0,57 & $-0,32$ & $-0,07$ & 0,29 & 1,06 & 0,00 & 1,06 & 5,76 \\
\hline Ingeniería & 0,42 & $-0,12$ & 0,22 & 0,11 & 0,02 & 0,26 & $-0,13$ & 0,91 & 5,03 \\
\hline Ciencias Medioambientales & $-0,03$ & 0,30 & 0,62 & 0,10 & 0,00 & 0,03 & 0,08 & 1,48 & 6,47 \\
\hline General & $-0,17$ & $-0,60$ & 2,50 & $-0,57$ & 1,33 & 1,00 & 0,14 & 1,67 & 32,03 \\
\hline Profesiones Sanitarias & 2,00 & 1,00 & $-0,83$ & 5,00 & 0,33 & 0,38 & $-0,82$ & 1,00 & 8,82 \\
\hline Inmunología y Microbiología & $-0,35$ & 0,27 & 0,36 & 0,21 & $-0,35$ & 0,73 & $-0,15$ & 0,29 & 11,12 \\
\hline Ciencia de Materiales & $-0,06$ & 0,21 & 0,24 & 0,36 & $-0,12$ & 0,07 & 0,03 & 0,87 & 7,67 \\
\hline Matemáticas & 0,19 & 0,32 & $-0,20$ & 0,36 & $-0,02$ & 0,12 & 0,14 & 1,15 & 2,99 \\
\hline Medicina & 0,41 & $-0,03$ & 0,51 & 0,29 & 0,14 & 0,24 & $-0,06$ & 2,56 & 5,05 \\
\hline Neurociencias & & 0,00 & $-1,00$ & & 0,50 & $-0,33$ & 2,50 & & 5 \\
\hline Enfermería & 0,00 & $-1,00$ & & & & $-1,00$ & & 1,00 & 3 \\
\hline Farmacología y Farmacia & 0,00 & 0,50 & $-0,33$ & 0,50 & 0,17 & 0,86 & $-1,00$ & $-1,00$ & 12,57 \\
\hline Física y Astronomía & $-0,17$ & 0,67 & 0,01 & 0,33 & $-0,09$ & 0,36 & $-0,03$ & 1,21 & 7,35 \\
\hline Psicología & 0,00 & 0,00 & $-0,50$ & 2,00 & 0,67 & 0,80 & 0,56 & 6,00 & 5,53 \\
\hline Ciencias Sociales & $-0,31$ & 0,22 & 0,82 & 1,25 & 0,38 & $-0,10$ & $-0,14$ & 2,69 & 4,84 \\
\hline Veterinaria & $-0,71$ & 0,50 & 2,33 & $-0,80$ & 3,00 & $-0,13$ & 0,43 & 0,43 & 5,27 \\
\hline
\end{tabular}

CpD: Citas por Documento

Fuente: SCImago Institutions Ranking

Biología Molecular, Medicina, Inmunología y Microbiología, Ciencias de la Decisión, y Ciencias Sociales.

Ingeniería es la primera en producción con 2287 documentos, el $16,12 \%$ del total institucional. Su visibilidad no se corresponde con su representatividad productiva, siendo la tercera en este aspecto con el $11,43 \%$ del total de citas.

Informática es la segunda en producción con 2122 publicaciones, $15 \%$ del total institucional, y un ratio de citas por documento de 3,29. Mejora a Ingeniería con un crecimiento de 1,35. Su visibilidad, como en el caso de Ingeniería, está por debajo de su rendimiento productivo, con menos del $7 \%$ de las citas recibidas por la UPV.

Matemáticas es la tercera área en producción absoluta. Sus 1702 trabajos suponen el $12 \%$ de la producción institucional, con un crecimiento del 1,15 . Su visibilidad, en cambio, apenas supone el $5 \%$ institucional, y tan sólo 2 citas por documento.
Agricultura, situada en cuarto puesto de producción y segundo en citación es una de las más representativas. El $10 \%$ de representatividad en producción se ve superado por el $11,8 \%$ de visibilidad. El número de citas por documento es de 8,36 y presenta una de las tasas de crecimiento más moderadas de 1,08. La UPV encabeza la producción de esta área entre las universidades politécnicas españolas, donde aglutina más del $40 \%$ tanto en producción como en citación.

Química, con 1182 publicaciones sitúa a la UPV en el puesto duodécimo del ranking nacional de universidades que proporciona el SIR, y supone el $8,33 \%$ del total institucional. El porcentaje de producción durante todo el período mantiene una tendencia positiva superior al $15 \%$ en todos los años excepto en 2003, lo que la convierte en un área muy consolidada. Es el área con mayor visibilidad de la UPV, con más del $20 \%$ de las citas absolutas. De acusado predominio también entre las politécnicas españolas, con más de la mitad de la 
producción y más del $70 \%$ de las citas en el área. Presenta, asimismo, el mayor número de citas por documento, 17,56.

En Ciencia de Materiales los 827 documentos representan el 5,8\% institucional, y la sitúan en el séptimo, puesto dentro de la UPV, superando la representatividad de sus dominios de referencia (España, $4 \%$, y Valencia, 3\%). Su crecimiento durante el período es de 0,87 , pasando de 70 trabajos en 2003 a 131 en 2010. El número de citas por documento es superior a la media 7,67 y recibe el $6,3 \%$ de las citas de la UPV.

Física, en sexta posición produce el 6,2\% de la UPV, muy similar a su visibilidad, $6,5 \%$. Su crecimiento de 1,21 y un número de citas por documento superior a la media le permite mantener una buena situación.

Ingeniería Química, octava con 748 publicaciones, un $5.3 \%$, y una tasa de crecimiento de 0,96 , se sitúa en el cuarto puesto de citación, 8,3\%, y una de las mayores tasas de citación con 11,24.

Biología Molecular solo representa el $4 \%$ de la producción de la UPV, y su visibilidad asciende al $7 \%$. Sin embargo, su característica principal es que la institución aporta el $44,3 \%$ de producción y el $42,5 \%$ de citación del total de trabajos publicados por las universidades politécnicas nacionales.

Medicina, un caso parecido al anterior, no es una de las más destacadas en el contexto institucional. Representa el 3,6\% de la producción de la UPV, y el $2,6 \%$ de su visibilidad. Además experimenta un crecimiento de 2,56 y un promedio de citas de 5,05 . Destaca, en cambio, cuando se compara con las otras universidades politécnicas. Es la primera en producción, con el $40 \%$ del total nacional y la segunda en citación con el 34\% de las citas.

Inmunología y Microbiología supone escasa producción a escala institucional, con una tasa de crecimiento de 0,29. En cambio, destaca entre las politécnicas tanto en producción como en visibilidad.

Ciencias de la Decisión es otra de las áreas poco representativas individualmente y muy influyente en su comparación con las otras universidades. Cuenta con una de las tasas de crecimiento más altas, cercana al $110 \%$ y 4,8 citas por documento, y aunque solo representa el $1,47 \%$ de la producción institucional, supone el $41 \%$ de producción y el $47,7 \%$ de citación entre las politécnicas nacionales.

Finalmente, Ciencias Sociales con 264 documentos y un crecimiento de 2,69, representa el 35\% del total de los trabajos del área y el $47 \%$ de la visibilidad de las universidades politécnicas.

\section{DISCUSIÓN}

La UPV se sitúa en el puesto 409 de las 3040 instituciones que componen el SIR World Report 2011 (SIR, 2011). Ocupa el puesto 19 de 1400 ins- tituciones en el SIR Ibero-American Ranking 2012 (SIR, 2012b), y el puesto 9 del ranking de universidades españolas en el mismo período analizado, solo por detrás de la Universitat de València que es la que lidera la producción de las universidades valencianas (Moya-Anegón y otros, 2011).

El crecimiento de la producción experimentado $(1,51)$, muy superior al de España y la Comunitat Valenciana $(0,84$ y 0.79$)$ puede estar motivado por varias causas. Por una parte, por la implicación de la UPV en las estructuras de investigación, y especialmente derivada de su participación en el desarrollo del Proyecto Campus de Excelencia Internacional (CEI). Por otra parte, gracias a la ampliación de la financiación proveniente de los programas de investigación nacionales y europeos que aumentan las posibilidades investigadoras de la Universidad (UPV, 2011b). Estudios recientes ponen de relieve que la UPV ha aumentado su esfuerzo en la realización de trabajos de investigación en colaboración con instituciones nacionales y extranjeras, principalmente universidades. La UPV mantiene relaciones de colaboración científica internacional con 665 instituciones, lo que supone un crecimiento del $60 \%$ en el número de instituciones colaboradoras respecto a los resultados de trabajos anteriores (Codina-Canet y Perianes, 2012). Este hecho debería repercutir positivamente no solo en el aumento de su producción, sino también en el de su visibilidad. Otro factor determinante es la propia exigencia del sistema de incentivos y promociones aplicado al personal investigador en la evaluación de su trayectoria profesional, puesto que el principal criterio para la obtención de reconocimiento, en forma de sexenios por ejemplo, son las publicaciones científicas, siendo mejor valoradas las indizadas en bases de datos internacionales.

Con respecto a la visibilidad, el empleo de la citación para describir la actividad científica debe considerar la ambigüedad que supone este indicador y tenerlo en cuenta en la interpretación de la realidad que describe. En este sentido, Sancho y Campanario identificaron patrones similares a los obtenidos por Garfield y McRoberts respecto a la parcialidad de los autores en el uso de la citación y la proporcionalidad de los artículos citados (Sancho, 1990; Campanario y otros, 1998). Por tanto, la frecuencia con que se cita un trabajo depende tanto del tamaño del área científica como de la calidad del trabajo. Por este motivo los trabajos de áreas minoritarias tienen mayores dificultades para alcanzar valores altos. Estas circunstancias han de tenerse en cuenta a la hora de comparar los datos de citación obtenidos en las distintas áreas temáticas. Así, en el caso de la UPV, se puede comprobar que las áreas más citadas no se corresponden necesariamente con las más productivas. Es el caso de Química con 20752 citas, que se sitúa en quinta posición en el ranking de producción, mostrando una diferencia significativa respecto a la tercera en citación, Ingeniería (11497), que ocupa a su vez el primer puesto en producción. 
Así, los resultados observados se encuentran dentro de lo esperado dado el perfil temático de la actividad de la UPV, y en consonancia con otros estudios en los que se constata el predominio de publicaciones con visibilidad internacional en disciplinas como la Química o la Tecnología (Bordons y otros, 2010). Estos datos, unidos a la peculiar idiosincrasia institucional en los hábitos de publicación, un tercio de los trabajos se difunden en forma de comunicaciones a congresos, y en la citación en las áreas técnicas, explican el retroceso de la visibilidad esperada derivado del análisis del indicador de impacto normalizado (ASSJR). En ese mismo sentido apunta la evolución del número de trabajos según el cuartil de la revista receptora. El aumento del $10 \%$ de trabajos en revistas de tercer y cuarto cuartil también puede afectar de forma determinante sobre la pérdida de visibilidad esperada detectada.

El análisis de la visibilidad, y a pesar del descenso del impacto esperado, presenta también lecturas positivas. Por un lado, el hecho de mantener en torno al $40 \%$ de trabajos publicados en revistas de primer cuartil a lo largo del período. Por otro, contar con visibilidades esperadas superiores a las obtenidas por las producciones de las principales politécnicas nacionales. Solo la Politécnica de Cartagena, cinco veces inferior a la UPV en producción y siete veces menor en citación, presenta un porcentaje mayor de trabajos en primer cuartil y, como se puede apreciar en la figura 4, es la única que supera a la Politécnica de Valencia en la evolución del impacto normalizado.

Por lo que respecta a las áreas de investigación, no resulta sorprendente que en una universidad politécnica sean las especialidades de Ingeniería, Informática y Matemáticas las predominantes desde el punto de vista de la producción. De igual forma, tampoco es singular que disciplinas del ámbito biomédico o ciencias básicas como Química o Física obtengan posiciones relevantes desde el punto de vista de la visibilidad. Tomando como base tanto los resultados de la evolución de la tasa de crecimiento (tabla $\mathrm{V}$ ), como los datos de producción y citación (anexos 1 y 2), hemos establecido una categorización de áreas temáticas: áreas consolidadas, áreas emergentes, áreas invariables y áreas con crecimiento negativo.

Entre las disciplinas consolidadas se encuentran: Ingeniería, Informática, Matemáticas, Agricultura, Química, Ciencia de Materiales, Física e Ingeniería Química.

Las áreas con proyección son: Energía, Ciencias Medioambientales, Medicina, Biología Molecular, Ciencias de la Decisión y Ciencias Sociales.

Disciplinas con escasa variación son: Empresa, Economía, Ciencias de la Tierra, Inmunología y Microbiología, Arte y Humanidades, Farmacología, Psicología y General.

Finalmente, las áreas con crecimiento negativo son Enfermería, Profesiones Sanitarias, Odontología, Neurociencias y Veterinaria.

Pero, además de esa categorización basada en la producción interna, es preciso destacar los rasgos distintivos de la UPV, revelados a partir de su comparación con el resto de universidades politécnicas nacionales (tabla IV). Reducido, pues, el universo de comparación a la producción estrictamente politécnica, la valenciana se erige como líder en producción y visibilidad en áreas como Agricultura, Biología Molecular, Química, Ciencias de la Decisión y Ciencias Sociales. Incluso en disciplinas de menor producción como Inmunología, Psicología, Odontología o Empresa obtiene valores entre el 40 y el $50 \%$ de la producción politécnica nacional.

Figura 4. Evolución del impacto normalizado. Universidades Politécnicas. 2003-2010

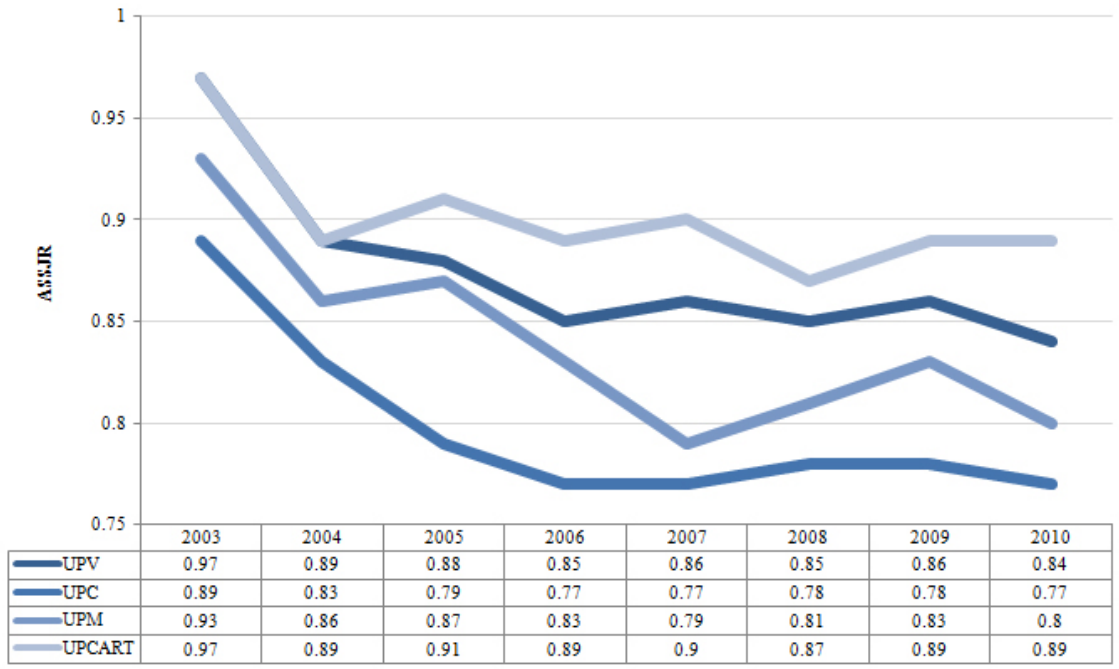


Especial mención merecen las áreas de Química e Ingeniería Química. La primera representa más de la mitad de la producción politécnica. Además, recibe más del $70 \%$ de las citas. Por su parte, la UPV es la segunda en producción en Ingeniería Química, pero recoge más de la mitad de las citas totales de la especialidad.

La UPV, por tanto, presenta rasgos distintivos interesantes cuando se compara con instituciones nacionales similares. Frente a la representatividad de las politécnicas de Cataluña o Madrid en áreas como Ingeniería, Informática, Matemáticas, Física, Ciencia de Materiales, Ciencias de la Tierra o Energía, disciplinas ligadas al habitual desempeño de investigaciones técnicas, la politécnica valenciana presenta representatividad y visibilidad orientada hacia disciplinas biomédicas (Biología Molecular, Inmunología, Agricultura, Medicina), por un lado, y a especialidades del ámbito de las Ciencias Sociales, aunque estas aún con producciones menos significativas (Economía, Empresa, Psicología, Ciencias de la Decisión, Ciencias Sociales), por otro.

La especialización temática representada en la figura 5 muestra como los dominios sobresalientes se corresponden con las áreas consolidadas y emergentes anteriormente descritas. La distribución temática en 2010 (color más claro), presenta una significativa evolución, en comparación con el mismo perfil en 2003. Las principales áreas incrementan su representatividad. Otras incipientes aumentan significativamente, como Medicina, Ciencias de la Decisión, Ciencias Sociales y Ciencias Medioambientales, con tasas de crecimiento elevadas $(2,56,3,85,2,69$ y 1,5 , respectivamente).
Estos resultados sobre especialización temática coinciden con hallazgos similares presentados en el estudio sobre caracterización de la actividad investigadora de la Universidad (UPV, 2011c). Este informe señala que las estructuras de investigación con mayor capacidad de producción científica fueron en 2008 el Instituto Universitario de Telecomunicación y Aplicaciones Multimedia, el Instituto Universitario de Automática e Informática Industrial, el Instituto Universitario de Aplicaciones de las Tecnologías de Información y Comunicaciones Avanzadas, el Departamento de Sistemas Informáticos y Computación y el Instituto Universitario Mixto de Tecnología Química (UPV-CSIC).

\section{CONCLUSIONES}

Se ha analizado la producción científica con visibilidad internacional de la UPV y su comparación con los agregados regional y nacional, además de con el resto de universidades politécnicas. Ello ha permitido la caracterización de la especialización temática y su evolución a lo largo del período analizado. De acuerdo con los objetivos planteados, y con los resultados obtenidos podemos concluir que:

En primer lugar, se constata un crecimiento interanual positivo decreciente de la producción científica excepto en los años impares al final del período. En este sentido, aunque el crecimiento de la UPV presenta una evolución más irregular que sus dominios geográficos de referencia durante los ocho años analizados, el crecimiento fue significativamente superior (en torno a los 70 puntos de diferencia).

Figura 5. Perfil temático de la producción. UPV 2003, 2010

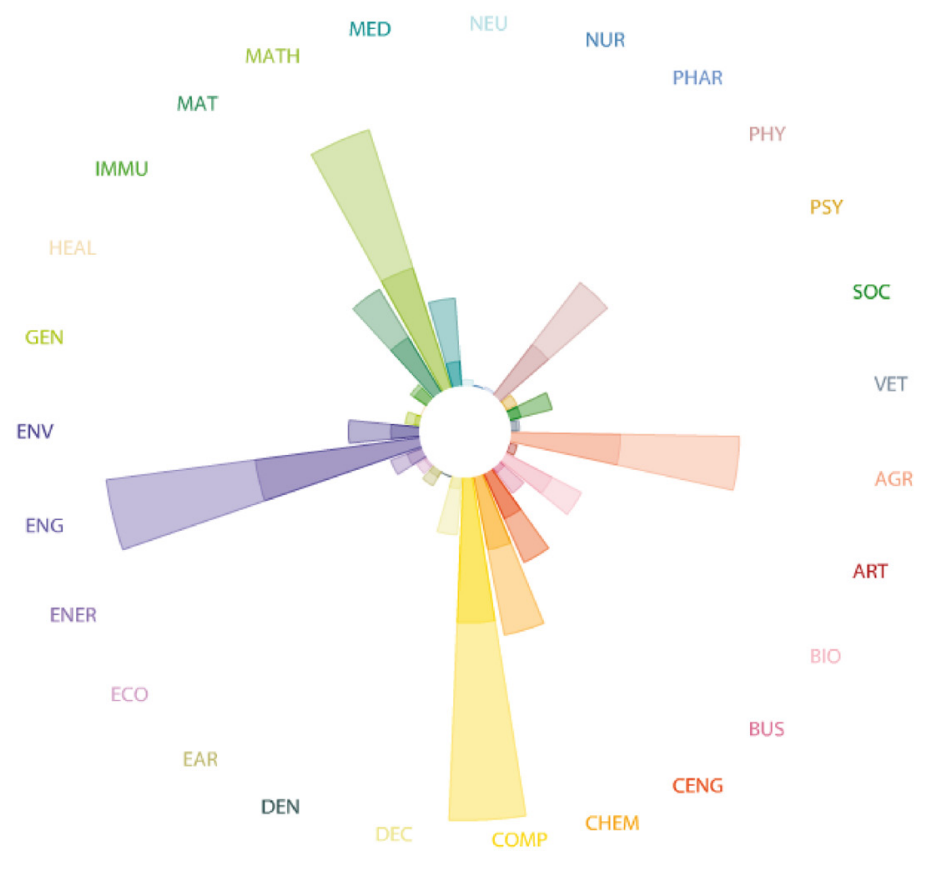


Por lo que respecta a la especialización, el análisis de las áreas temáticas ha permitido conocer las disciplinas más destacadas y establecer la caracterización científica de la UPV. Más de la mitad de la producción institucional se adscribe a las áreas de Ingeniería, Informática, Química y Matemáticas, mostrándose como las principales áreas del devenir científico institucional, y situando a la UPV en el segundo eje del Triángulo de la Ciencia. Además, un tercio de los trabajos se publican en forma de comunicaciones a congresos. Si a ello añadimos los publicados en forma de artículos, ambas tipologías representan el $95 \%$ de la producción. A su vez, el $95 \%$ de los trabajos se publican en inglés y reciben más del $99,5 \%$ de las citas totales. Por otro lado, el análisis de las distintas áreas temáticas ha permitido su clasificación, basada en el crecimiento y la representatividad institucional:

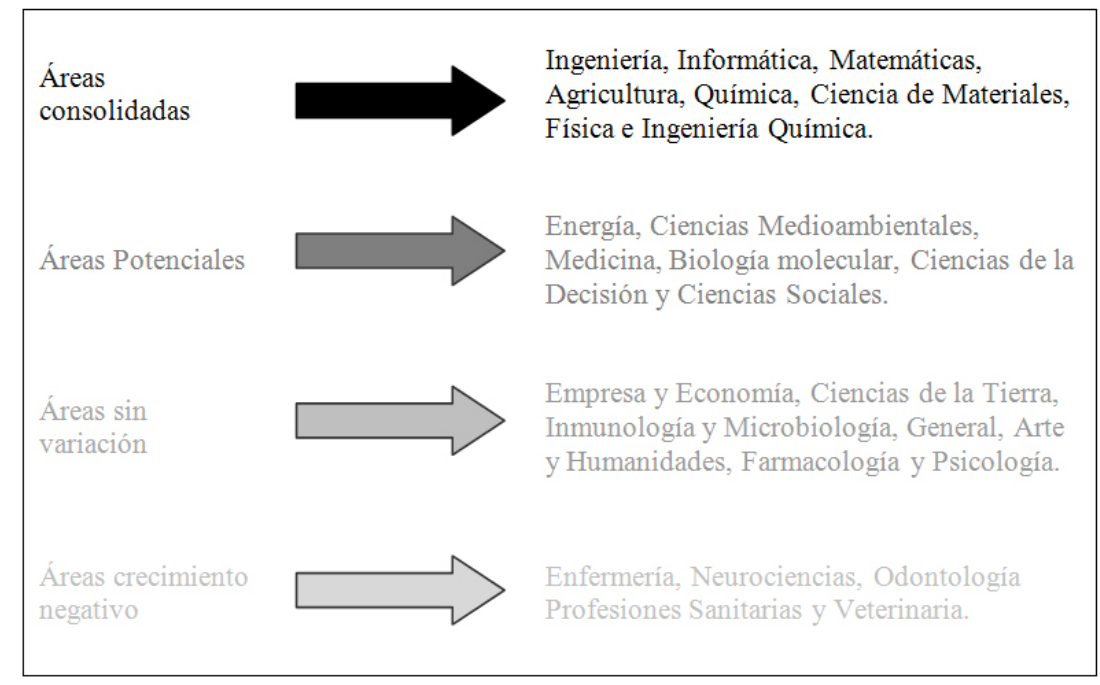

Sobre su posición respecto a instituciones afines, la UPV es la segunda politécnica española en producción y citación absolutas, por detrás de la de Cataluña. Mantiene una posición predominante en Agricultura, Biología Molecular, Química, Ciencias de la Decisión, Ciencias Sociales y Química. En este sentido, aunque los principales dominios según su producción son de carácter politécnico, son otras áreas menos técnicas las que presentan mayor representatividad y visibilidad, evidenciando señas de identidad distintivas respecto de sus homólogas. Esa identidad diferenciadora que en principio puede resultar llamativa, puede explicarse por el trabajo de investigación que desarrollan centros e institutos asociados como el Instituto Universitario Mixto de Tecnología Química (UPV-CSIC), el Instituto Universitario de Bioingeniería y Tecnología Orientada al Ser Humano, el Instituto Universitario de Ingeniería de Alimentos para el Desarrollo, el Instituto Universitario Mixto de Biología Molecular y Celular de Plantas (UPV-CSIC), el Instituto de Reconocimiento Molecular y Desarrollo Tecnológico, el Instituto de Biomecánica de Valencia, el Centro de Biomateriales o el Centro de Investigación en Gestión de Empresas, entre otros. Los trabajos en ellos desarrollados ayudan a fortalecer el arraigo de áreas biomédicas, agrícolas y de ciencias sociales, disciplinas tradicionalmente distantes en instituciones de este tipo.

En lo concerniente al impacto de sus trabajos, el retroceso de la visibilidad esperada se corresponde con un aumento de los trabajos publicados

en revistas de tercer y cuarto cuartil. A pesar de este descenso, la UPV mantiene hasta un $40 \%$ de trabajos publicados en revistas de primer cuartil y cuenta con visibilidades esperadas superiores a las obtenidas por las principales politécnicas nacionales. En este sentido, es preciso mencionar que las universidades politécnicas parten con cierta desventaja respecto a las generalistas. Las disciplinas técnicas no tienen tan asentado el sistema de publicación de resultados en revistas científicas ni, por ende, el de citación, especialmente si se compara con instituciones en las que predominan las áreas médicas o de ciencias básicas.

Finalmente, es importante destacar la necesidad de realizar trabajos futuros que permitan profundizar en algunos de los hallazgos expuestos, especialmente aquellos que posibiliten la desagregación y caracterización del papel que juegan las subestructuras institucionales (institutos, centros, departamentos, etc.). Esto ayudaría a explicar con mayor precisión la importancia creciente de disciplinas biomédicas y de ciencias sociales en la institución, o el papel relevante de la Química como especialidad destacada en producción y visibilidad. También resultarían interesantes aquellos estudios dirigidos al análisis e influencia de la colaboración científica, por tratarse de un importante fenómeno en crecimiento y que afecta a procesos críticos en el desarrollo de una institución investigadora. O estudios comparativos que ampliaran la comparación entre universidades politécnicas en este trabajo iniciada. 


\section{BIBLIOGRAFÍA}

Alonso-Arroyo, A.; Pulgarín, A.; Gil-Leiva, I. (2006). Análisis bibliométrico de la producción científica de la Universidad Politécnica de Valencia 1973-2001. Revista Española de Documentación Científica, 29 (3), p. 349-355.

Alonso-Arroyo, A.; Pulgarín, A.; Gil-Leiva, I. (2005). Estudio cienciométrico de la colaboración científica en la Universidad Politécnica de Valencia. Information Research 11(1) paper 245 [Disponible: http: //InformationR.net/ir/11-1/paper245.html]. [Consulta: 29/06/2012].

Bar-Ilan, J. (2010). Citations to the 'Introduction to infometrics 'indexed by WOS, Scopus and Google Scholar. Scientometrics, 82 (3), p. 495-506.

Bordons, M.; Morillo, F.; Sancho R.; Gómez, I. (2010). Estructura y especialización de las universidades. En: Sanz Menéndez, L; Cruz Castro, L. Análisis sobre Ciencia e innovación en España. Madrid: FECYT. P. 151-183.

Bosman J.; van Mourik I.; Rasch M.; Sieverts E.; Verhoeff H. (2006). Scopus reviewed and compared. Utrech: University Library. [Disponible: http://igiturarchive.library.uu.nl/DARLIN/2006-1220-200432/ Scopus\%20doorgelicht\%20\&\%20vergeleken\%20 -\%20translated.pdf]. [Consulta: 29/06/2012].

Campanario, J.M.; Cabos, W.; Hidalgo, M.A. (1998). El impacto de la producción científica de la Universidad de Alcalá de Henares. Revista Española de Documentación Científica, 121 (4), p. 402-405.

Codina-Canet, M.A.; Perianes-Rodríguez, A. (2012). Análisis de la colaboración científica de la Universidad Politécnica de Valencia (Scopus, 2003-2008). Métodos de Información, II, 3 (4), p. 87-105. [Disponible: http://dx.doi.org/10.5557/IIMEI2N2-087105]. [Consulta: 29/06/2012].

Escalona Fernández, M. I. ; Lagar Barbosa, P.; Pulgarín Guerrero, A. (2010). Web of Science vs. SCOPUS: un estudio cuantitativo en Ingeniería Química. Anales de Documentación, 13, p. 159-175.

González-Pereira, B.; Guerrero-Bote, V.P.; Moya-Anegón, F. (2009). The SJR indicator: A new indicator of journals' scientific prestige. Madrid: SCImago Research Group [Disponible: http://arxiv.org/ftp/ arxiv/papers/0912/0912.4141.pdf]. [Consulta: 29/06/2012].

Goodman, D.; Deis, L. (2005). Web of Science (2004 version) and Scopus. The Charleston Advisor, 6 (3), p. 5-21.

Herrero Solana, V.; Vargas Quesada, B. (2010). Especialización temática de la producción científica. En: Sanz Menéndez, L; Cruz Castro, L. Análisis sobre Ciencia e innovación en España. Madrid: FECYT.

LaGuardia, C. (2005). E-Views and Reviews: Scopus vs. Web of Science. Library Journal, January. [Dis- ponible: http://www.libraryjournal.com/article/ CA491154.html]. [Consulta: 29/06/2012].

Leydesdorff, L. (2012). World shares of publications of the USA, EU-27, and China compared and predicted using the new Web of Science interface versus Scopus. El Profesional de la Información, 21 (1), p. 43-49.

Maltrás, B. (2003). Los indicadores bibliométricos: Fundamentos y aplicación al análisis de la ciencia. Gijón: Trea.

Moya-Anegón, F.; Chinchilla-Rodríguez, Z.; BenaventPérez, M.; Corera-Álvarez, E. ; González-Molina, A. ; Vargas-Quesada, B. (2011). Indicadores bibliométricos de la actividad científica española 2008. Madrid: FECYT.

Reyes Davó, E.; Fernández de Lucio, I. (1994). La investigación y el desarrollo tecnológico en la Universidad Politécnica de Valencia. Política Científica, 39, p. 41-44.

Sancho, R. (1990). Indicadores bibliométricos utilizados en la evaluación de la ciencia y la tecnología. Revisión bibliográfica. Revista Española de Documentación Científica, 13 (3-4), p. 842-865.

Sancho, R. (2002). Indicadores de los sistemas de ciencia, tecnología e innovación. Economía industrial, 343 (1), p. 97-109.

SIR. (2011). SIR World Report 2011. [Disponible: http://www.scimagoir.com/pdf/sir_2011_world_ report.pdf]. [Consulta: 29/06/2012].

SIR. (2012a). SCImago Institutions Ranking. [Disponible: http://www.scimagoir.com/methodology. php]. [Consulta: 29/06/2012].

SIR. (2012b). Ranking Iberoamericano 2012. [Disponible: http://www.scimagoir.com/pdf/ranking iberoamericano_2012.pdf]. [Consulta: 29/06/2012].

Universidad Politécnica de Valencia. (2007). Plan estratégico UPV. Valencia: MASmedios. [Disponible: http://planestrategico.upv.es/wp-admin/ descargas/plan estrategico.pdf]. [Consulta: 29/06/2012].

Universidad Politécnica de Valencia. (2011a). Valencia Campus de Excelencia Internacional. [Disponible: http://www.vlc-campus.com/wp-content/documents/VLC_Memoria.pdf]. [Consulta: 29/06/2012].

Universidad Politécnica de Valencia. (2011b). Memoria 2009. Valencia: Vicerrectorado de Investigación de la UPV. [Disponible: http://www.upv.es/upl/ U0553058.pdf]. [Consulta: 29/06/2012].

Universidad Politécnica de Valencia. (2011c). Valoración de la actividad investigadora personalizada de Estructuras y Grupos de Investigación de la UPV en el año 2008. Valencia: Vicerrectorado de Investigación de la UPV. 


\section{ANEXOS}

Anexo I. Producción Científica. Áreas Temáticas. UPV 2003-2010

\begin{tabular}{|c|c|c|c|c|c|c|c|c|c|}
\hline Área Temática & 2003 & 2004 & 2005 & 2006 & 2007 & 2008 & 2009 & 2010 & Total \\
\hline Agricultura & 119 & 103 & 135 & 164 & 207 & 217 & 230 & 248 & 1423 \\
\hline Arte y Humanidades & 1 & 2 & 0 & 1 & 5 & 4 & 8 & 9 & 30 \\
\hline Biología Molecular & 57 & 55 & 51 & 68 & 84 & 80 & 106 & 93 & 594 \\
\hline Empresa & 7 & 9 & 4 & 21 & 15 & 28 & 35 & 35 & 154 \\
\hline Ingeniería Química & 54 & 86 & 97 & 106 & 99 & 85 & 115 & 106 & 748 \\
\hline Química & 81 & 133 & 161 & 159 & 158 & 145 & 170 & 175 & 1182 \\
\hline Informática & 158 & 200 & 231 & 204 & 292 & 296 & 369 & 372 & 2122 \\
\hline Ciencias de la Decisión & 13 & 12 & 18 & 25 & 27 & 41 & 45 & 63 & 244 \\
\hline Odontología & 0 & 0 & 1 & 0 & 0 & 0 & 2 & 2 & 5 \\
\hline Ciencias de la Tierra & 8 & 16 & 18 & 11 & 15 & 21 & 26 & 19 & 134 \\
\hline Economía & 4 & 0 & 2 & 6 & 5 & 12 & 9 & 14 & 52 \\
\hline Energía & 18 & 14 & 22 & 15 & 14 & 18 & 37 & 37 & 175 \\
\hline Ingeniería & 180 & 255 & 224 & 274 & 305 & 312 & 394 & 343 & 2287 \\
\hline Ciencias Medioambientales & 31 & 30 & 39 & 63 & 69 & 69 & 71 & 77 & 449 \\
\hline General & 6 & 5 & 2 & 7 & 3 & 7 & 14 & 16 & 60 \\
\hline Profesiones Sanitarias & 1 & 3 & 6 & 1 & 6 & 8 & 11 & 2 & 38 \\
\hline Inmunología y Microbiología & 17 & 11 & 14 & 19 & 23 & 15 & 26 & 22 & 147 \\
\hline Ciencia de Materiales & 70 & 66 & 80 & 99 & 135 & 119 & 127 & 131 & 827 \\
\hline Matemáticas & 137 & 163 & 215 & 172 & 234 & 230 & 257 & 294 & 1702 \\
\hline Medicina & 27 & 38 & 37 & 56 & 72 & 82 & 102 & 96 & 510 \\
\hline Neurociencias & 0 & 1 & 1 & 0 & 2 & 3 & 2 & 7 & 16 \\
\hline Enfermería & 1 & 1 & 0 & 0 & 0 & 1 & 0 & 2 & 5 \\
\hline Farmacología y Farmacia & 4 & 4 & 6 & 4 & 6 & 7 & 13 & 0 & 44 \\
\hline Física y Astronomía & 70 & 58 & 97 & 98 & 130 & 118 & 160 & 155 & 886 \\
\hline Psicología & 2 & 2 & 2 & 1 & 3 & 5 & 9 & 14 & 38 \\
\hline Ciencias Sociales & 13 & 9 & 11 & 20 & 45 & 62 & 56 & 48 & 264 \\
\hline Veterinaria & 7 & 2 & 3 & 10 & 2 & 8 & 7 & 10 & 49 \\
\hline TOTAL & 1086 & 1278 & 1477 & 1604 & 1956 & 1993 & 2401 & 2390 & 14185 \\
\hline
\end{tabular}

Fuente: SCImago Institutions Ranking 


\section{ANEXOS}

Anexo II. Citación. Áreas Temáticas. UPV 2003-2010

\begin{tabular}{|c|c|c|c|c|c|c|c|c|c|}
\hline Área Temática & 2003 & 2004 & 2005 & 2006 & 2007 & 2008 & 2009 & 2010 & Total \\
\hline Agricultura & 2251 & 1564 & 1965 & 1731 & 1606 & 1395 & 980 & 405 & 11897 \\
\hline Arte y Humanidades & 0 & 30 & 0 & 11 & 13 & 10 & 24 & 4 & 92 \\
\hline Biología Molecular & 1589 & 790 & 1292 & 925 & 880 & 736 & 520 & 275 & 7007 \\
\hline Empresa & 71 & 24 & 23 & 180 & 80 & 118 & 45 & 39 & 580 \\
\hline Ingeniería Química & 1423 & 1833 & 1269 & 1236 & 992 & 744 & 693 & 219 & 8409 \\
\hline Química & 4132 & 3182 & 3622 & 3731 & 2691 & 1606 & 1208 & 580 & 20752 \\
\hline Informática & 617 & 1206 & 1475 & 824 & 1049 & 997 & 583 & 221 & 6972 \\
\hline Ciencias de la Decisión & 176 & 130 & 378 & 202 & 328 & 327 & 123 & 63 & 1727 \\
\hline Odontología & 0 & 0 & 10 & 0 & 0 & 0 & 3 & 0 & 13 \\
\hline Ciencias de la Tierra & 83 & 159 & 110 & 85 & 40 & 51 & 78 & 13 & 619 \\
\hline Economía & 72 & 0 & 1 & 108 & 19 & 46 & 27 & 10 & 283 \\
\hline Energía & 233 & 105 & 194 & 111 & 100 & 86 & 129 & 50 & 1008 \\
\hline Ingeniería & 1590 & 2045 & 1997 & 1763 & 1497 & 1392 & 944 & 269 & 11497 \\
\hline Ciencias Medioambientales & 584 & 407 & 368 & 383 & 470 & 335 & 254 & 106 & 2907 \\
\hline General & 71 & 423 & 15 & 678 & 25 & 239 & 414 & 57 & 1922 \\
\hline Profesiones Sanitarias & 12 & 178 & 45 & 5 & 27 & 46 & 22 & 0 & 335 \\
\hline Inmunología y Microbiología & 286 & 250 & 308 & 236 & 249 & 152 & 110 & 44 & 1635 \\
\hline Ciencia de Materiales & 1104 & 896 & 776 & 821 & 1204 & 753 & 594 & 197 & 6345 \\
\hline Matemáticas & 480 & 608 & 1198 & 662 & 925 & 746 & 308 & 167 & 5094 \\
\hline Medicina & 524 & 390 & 229 & 360 & 281 & 479 & 249 & 62 & 2574 \\
\hline Neurociencias & 0 & 13 & 4 & 0 & 4 & 59 & 0 & 0 & 80 \\
\hline Enfermería & 8 & 7 & 0 & 0 & 0 & 0 & 0 & 0 & 15 \\
\hline Farmacología y Farmacia & 97 & 61 & 120 & 74 & 67 & 84 & 50 & 0 & 553 \\
\hline Física y Astronomía & 1008 & 973 & 1099 & 840 & 1069 & 661 & 635 & 227 & 6512 \\
\hline Psicología & 23 & 48 & 32 & 9 & 63 & 22 & 8 & 5 & 210 \\
\hline Ciencias Sociales & 89 & 92 & 237 & 158 & 298 & 291 & 67 & 47 & 1279 \\
\hline Veterinaria & 88 & 17 & 60 & 59 & 2 & 19 & 10 & 3 & 258 \\
\hline TOTAL & 16611 & 15431 & 16827 & 15192 & 13979 & 11394 & 8078 & 3063 & 100575 \\
\hline
\end{tabular}

Fuente: SCImago Institutions Ranking 


\section{ANEXOS}

Anexo III. Visibilidad de la producción por cuartiles e impacto normalizado. UPV 2003-2010

\begin{tabular}{llllll}
\hline & ASSJR & Q4 & Q3 & Q2 & Q1 \\
\hline 2003 & 0,97 & 68 & 161 & 344 & 394 \\
\hline 2004 & 0,89 & 78 & 200 & 422 & 462 \\
\hline 2005 & 0,88 & 54 & 247 & 482 & 544 \\
\hline 2006 & 0,85 & 72 & 268 & 503 & 583 \\
\hline 2007 & 0,86 & 215 & 400 & 627 & 635 \\
\hline 2008 & 0,85 & 184 & 355 & 504 & 573 \\
\hline 2009 & 0,86 & 280 & 426 & 522 & 843 \\
\hline 2010 & 0,84 & 259 & 411 & 515 \\
\hline
\end{tabular}

Fuente: SCImago Institutions Ranking 